\title{
Nuevas Perspectivas para la Enseñanza de la Resolución de Problemas de Cálculo a Estudiantes de Ingeniería
}

\author{
New Perspectives for Teaching Calculus Problem Solving \\ to Engineering Students
}

\author{
William Becerra Salamanca, Norma Constanza Sarmiento Benavides \\ Departamento de Matemáticas, Universidad Militar Nueva Granada, Bogotá, Colombia \\ william.becerra@unimilitar.edu.co \\ norma.sarmiento@unimilitar.edu.co
}

\begin{abstract}
Resumen - En este artículo se presenta una reflexión en el campo de la Educación Matemática que a partir del análisis de los resultados de una investigación realizada por los autores en la Universidad Militar Nueva Granada (2013-2014), propone la exploración de nuevas rutas para el tratamiento de la Resolución de Problemas de Cálculo con estudiantes de ingeniería. Los nuevos enfoques pretender ir más allá de los tradicionales tratamientos Heurísticos y Metacognitivos surgidos especialmente de las escuelas de G. Polya y A. Schoenfeld, que caracterizaron la mencionada investigación y que, aunque universalmente han tenido gran vigencia, ameritan ya una revisión acorde con los tiempos actuales, con el surgimiento de nuevos problemas y ante la posibilidad de contar con recursos tecnológicos que cambian el panorama de la Educación Matemática en la formación de ingenieros.
\end{abstract}

Palabras clave-Educación Matemática - Enseñanza del Cálculo Heurísticas - Modelación - Resolución de Problemas Matemáticos - Sistemas de Representación.

Abstract-In this article a reflection is presented on the field of Mathematical Education which starting from the results analysis of an investigation made by the authors at the Universidad Militar Nueva Granada (2013-2014), whose purpose was the exploration of new ways for the treatment of the Calculus Problems Solving with engineering students. The new approaches pretend to go further the traditional Heuristic and Metacognitive treatments emerged specially from the Polya and Schoenfeld theories, which characterized the previously mentioned investigation and that, even though it has universally had great validity, they already deserve a review according to the present times, with the emergence of new problems and in the face of counting with technological resources that change the Mathematical Education panorama in the training of engineers.

Key Word - Calculus - Calculus Teaching - Heuristics Mathematical Problem Solving - Modelling - Representation Systems

Fecha de Recepción: 10 de noviembre de 2015

Fecha de Aceptación: 13 de junio de 2017

\section{INTRODUCCIÓN}

La sociedad actual experimenta un creciente desarrollo científico y los estudiantes necesitan estar preparados para comprender esa dinámica. En ese sentido, el contar con una buena formación matemática es una necesidad evidente particularmente en el caso de los estudiantes de ingeniería, cuya profesión se fundamenta en las Ciencias Básicas y se proyecta a su aplicación para resolver problemas y satisfacer necesidades de la sociedad.

La Resolución de Problemas (RP) desde hace más de tres décadas ha sido considerada como una de las actividades más importantes en el aprendizaje de las Matemáticas [3] y ha ocupado un lugar destacado de la investigación y el desarrollo de la Educación en este campo. Su tratamiento requiere especial atención para lograr que los estudiantes adquieran una mayor capacidad para transferir los conocimientos matemáticos de manera eficaz a la práctica.

La experiencia de los investigadores en la enseñanza del Cálculo en programas de ingeniería, y la abundante investigación sobre el tema, dan cuenta de la gran dificultad que representa para los estudiantes plantear y resolver problemas no rutinarios, es decir, problemas en los cuales la solución no se obtiene por aplicación directa de fórmulas, algoritmos o procedimientos estandarizados. Es frecuente que en los exámenes en que se pide resolver problemas, el espacio destinado para hacerlo quede en blanco. Es motivo de preocupación comprobar en la experiencia cotidiana los altos índices de fracaso de los estudiantes al intentar aplicar sus conocimientos a la solución de problemas.

Es muy importante entender que el desarrollo de las ideas matemáticas conlleva un proceso de reflexión donde el estudiante transforma su forma de pensar en la medida en que participa en una comunidad de práctica. que desarrolle 
recursos, estrategias y herramientas que le permitan superar dificultades o "atascos" iniciales y robustecer su forma de pensar matemáticamente [16].

El propósito central de este artículo de reflexión es el de presentar nuevas perspectivas para el tratamiento de la Resolución de Problemas en los cursos de Cálculo que se dictan en Ingeniería, a partir del análisis y revisión de una investigación ya realizada por los autores cuyos resultados han permitido detectar vacíos en la enseñanza tradicional de estos temas e invitan a la exploración de nuevas rutas.

La investigación en Resolución de Problemas por su parte enfrenta también sus propias dificultades y controversias. Por ejemplo, en [13] se afirma que realmente esta problemática se ha eludido hábilmente por la comunidad académica, la presencia de la RP no ha sido intensa y ha estado separada del desarrollo curricular. Por otra parte se admite que un gran número de propuestas en el ámbito internacional han establecido la RP como el eje central de la organización de los contenidos .En [16] se afirma que la investigación en RP carece de mecanismos eficientes de acumulación y discusión de los avances a nivel internacional, y como consecuencia de ello, muchos temas se reciclan o simplemente se designan con otros nombres.

\section{LA INVESTIGACIÓN}

La reflexión que se presenta en este artículo está relacionada con la investigación titulada Propuesta Didáctica enfocada a mejorar el desempeño de los estudiantes al plantear y resolver problemas de optimización, que se realizó durante el año 2013 y comienzos del 2014 en la Universidad Militar Nueva Granada. con estudiantes de Cálculo Diferencial de segundo semestre de ingeniería.

Son ampliamente reconocidas en el ámbito de la Educación Matemática las dificultades que tienen los estudiantes para plantear y resolver problemas No Rutinarios. En este sentido el proyecto se realizó con el objetivo de comprender y caracterizar las mencionadas dificultades y presentar una propuesta para mejorar el desempeño de los alumnos. Dicha propuesta se estructuró con dos ejes principales: primero, con un énfasis marcado en el empleo de estrategias Heurísticas y Metacognitivas [1].[2], y en segundo lugar, con base en la teoría de Registros de Representación Semiótica [5].

\section{A. Antecedentes}

Entre los temas que han sido centrales para la Educación Matemática en los últimos 30 años están la Resolución de Problemas, la Modelación matemática y, en general, las aplicaciones en otras disciplinas [6]. Particularmente durante los últimos diez años, el número de contribuciones a la investigación en este campo ha aumentado considerablemente y al abordar la cuestión de cómo adquirimos los conocimientos matemáticos, es difícil eludir el papel de su relación con los problemas del mundo real.

El estudio de las Matemáticas comprende mucho más que la memorización de reglas y fórmulas, fundamentalmente tiene que ver con la búsqueda de la verdad [14], con la comprensión del mundo. Se resalta la importancia de lograr que los estudiantes se planteen preguntas y busquen formas de resolverlas, que formulen conjeturas, empleen distintas representaciones, argumenten matemáticamente y desarrollen habilidades para comunicar sus resultados [13]. Un aspecto muy importante en el aprendizaje de las Matemáticas tiene que ver con la necesidad de que los estudiantes puedan utilizar eficientemente sus conocimientos en un contexto o en la solución de nuevos problemas.

George Polya realizó durante muchos años un importante trabajo dirigido a proporcionar elementos para que los estudiantes tuvieran mayor probabilidad de éxito al resolver problemas. Para tal efecto, desarrolló una serie de estrategias heurísticas [1] que, si bien no garantizan que al usarlas el problema quede resuelto, sí constituyen una guía que empleada correctamente puede contribuir a desarrollar en el estudiante hábitos eficientes de reflexión y estudio que a la larga rendirán sus frutos. En [1] se organizan las mencionadas estrategias heurísticas en cuatro fases: Primero, comprender el problema, viendo con claridad lo que se pide. Segundo, captar las relaciones para trazar un plan. Tercero, ejecutar el plan. Cuarto, volver atrás para revisar y discutir la solución encontrada. Dando continuidad a los planteamientos de su obra más representativa How to Solve it cuya primera edición data de 1945 siendo la más reciente una de 2013, en [12] formuló los principios del Razonamiento Plausible que, en su concepto, es la única forma de razonamiento que utilizamos en la vida cotidiana. El conocimiento matemático se certifica mediante las demostraciones, pero para aprender algo nuevo y para resolver problemas necesitamos el Razonamiento Plausible. Se trata de intuir, de formular conjeturas, de ensayar. Hay que intuir un teorema matemático antes de probarlo, hay combinar observaciones, seguir analogías y probar una y otra vez.

Alan Schoenfeld, reconocido científico internacional propone con base en sus investigaciones, un marco de referencia en el cual las estrategias heurísticas son sólo uno de los componentes. En [2], las complementa en un modelo que incluye los siguientes factores: el conocimiento de base, los aspectos meta cognitivos o de control, los aspectos afectivos y la comunidad de práctica. En su concepto [2], no es suficiente con que el alumno conozca estrategias, es tal vez más importante que participe en experiencias relacionadas con el cuándo y cómo utilizarlas.

En [3] se plantea que cuando los problemas se tratan en contextos específicos como los de los libros de texto, el conocimiento de la materia tiene un papel determinante, pero cuando los problemas no hacen referencia a situaciones tan familiares para el alumno, la presencia de estrategias se hace más importante para llegar a la solución. 
Las habilidades cognitivas requeridas para resolver problemas están relacionadas con el desarrollo del pensamiento de alto nivel. Puede afirmarse [3] que la mayoría de ellas incluyen:

- Un pensamiento no algorítmico.

- Que el individuo considere varias formas de solución.

- Considerar el uso de diversos criterios, algunos de los cuales pueden estar en contradicción.

- Un pensamiento que maneje la incertidumbre.

- Un pensamiento que incluya mecanismos de monitoreo.

Después de más de dos décadas de trabajo y la obtención de la Medalla Félix Klein por los logros de toda su vida, en [7] Schoenfeld actualiza su mirada sobra la RP. El libro se centra en cómo y por qué las personas toman las decisiones que toman al participar en actividades intensivas de conocimiento; ofrece un relato teórico de las decisiones que los maestros toman en medio de la extraordinaria complejidad de las interacciones en el aula.

En 1985 cuando escribió [2], consideraba que esas categorías eran necesarias y suficientes para explicar el éxito o el fracaso en la solución de problemas. En efecto, han soportado el paso de los años por más de un cuarto de siglo pero, si bien estas categorías definían un marco para examinar la RP, no constituían propiamente una teoría. No permitían explicar cómo y por qué los solucionadores de problemas se basaban en conocimientos o empleaban estrategias específicas. Ese es el enfoque de su nuevo trabajo que adicionalmente pretende que esta teoría sea aplicable a la Física y a otros dominios de conocimiento. Es decir, su objetivo es el de configurar una teoría de la toma de decisiones.

En relación con los registros de representación semiótica, en [5] se afirma que muchos estudiantes perciben una gran distancia entre las formas del pensamiento matemático y las formas de pensar fuera de las Matemáticas y sugiere realizar investigaciones sobre los procesos cognitivos vinculados. En el marco de la teoría de Duval, la actividad matemática se realiza en un contexto de representación que requiere una coordinación interna. Las dos principales transformaciones de representaciones son el tratamiento y la conversión pues ellas "yacen en el corazón de la actividad matemática y son un todo en la resolución de problemas". La dificultad que la mayoría de los estudiantes tiene para realizar estas transformaciones es tan profunda que la conversión puede considerarse como el umbral de la comprensión [9]. Este proceso podría verse como "un truco difícil de aprender y que además no se enseña".

Desde otra perspectiva, en [10] se plantea una mirada a cerca de la importancia de la Comprensión como objetivo de todo proyecto educativo que, en cierto sentido, es análoga a la que Duval establece en su teoría de los Registros de Representación Semiótica. En efecto, en concepto de Gardner, una persona sólo puede comprender bien un concepto - y dar muestras convincentes de su comprensión - si puede desarrollar múltiples representaciones de sus aspectos esenciales. Además, es conveniente que estas representaciones se basen en varios sistemas de símbolos, esquemas, marcos de referencia e inteligencias: yendo más allá de las analogías - en realidad, tomando la dirección contraria - las representaciones podrán ser más exactas y exhaustivas. Y dado que cada representación destaca necesariamente ciertos aspectos del tema por encima de los demás, el objetivo final es sintetizar las diversas representaciones de la manera más exhaustiva posible.

Esta afirmación, sostiene Gardner, tiene varias consecuencias educativas. En primer lugar, es necesario dedicar el tiempo suficiente a un tema. En segundo lugar, es esencial describir el tema de varias maneras, tanto para ilustrar sus complejidades como para que llegue a todos los estudiantes. En tercer lugar, es muy conveniente que estos distintos enfoques se dirijan explícitamente a una gama de inteligencias, aptitudes e intereses.

Lo anterior no significa que se deba bombardear a los alumnos con cualquier tipo concebible de material con la esperanza de abarcar todo su abanico de capacidades. En este sentido hay que rescatar el aspecto artesanal de la pedagogía, que no se presta a un enfoque algorítmico. El profesor debe tomar decisiones para elegir los enfoques y tratamientos más pertinentes para cada tema [10].

Surge para el docente la oportunidad de volver a examinar los temas y encontrar nuevas formas de transmitir sus aspectos esenciales, de la manera más clara y precisa posible, a unas mentes distintas.

\section{B. Metodología}

Desde el punto de vista metodológico se trata de un estudio de caso instrumental por cuanto su finalidad es comprender las circunstancias que rodean la implementación de una propuesta de enseñanza con el propósito de afinar la mirada y revisar las prácticas habituales sin la pretensión de formular generalizaciones de gran dimensión. Por la naturaleza de los objetos matemáticos abordados, se inscribe en la Línea de Pensamiento Matemático Avanzado [8] por cuanto hace referencia a procesos en los cuales hay un fuerte componente de abstracción que consiste en la sustitución de fenómenos concretos por conceptos que están en la mente. No puede decirse que la abstracción sea exclusiva de las matemáticas superiores [11]. La comprensión de los conceptos matemáticos tiene que ver con la riqueza y flexibilidad de las representaciones mentales que se tienen de ellos y una de las razones de su complejidad es que ellos pueden jugar el papel tanto de procesos como de objetos.

La idea es tratar de cambiar una cierta ilusión de transparencia o autoevidencia que algunos educadores atribuyen a los objetos matemáticos, la organización de los libros de texto y de muchas clases parecen basarse en que los conceptos se adquieren mediante su definición y que con sólo conocerla los estudiantes podrán realizar conexiones múltiples y resolver problemas 
[11].La investigación se enfoca en tratar de comprender mejor las dificultades que tienen los estudiantes para diseñar estrategias que les ayuden a mejorar.

En este caso se trata de problemas expresados en palabras en los cuales entran en juego los conceptos básicos del Cálculo Diferencial en situaciones de optimización. Problemas en las cuales la solución no es inmediata ni puede hallarse por aplicación directa de fórmulas o procedimientos estandarizados.

En desarrollo del proyecto se realizaron cuatro acciones específicas. Son ellas:

$$
A_{1} \text { : Prueba Piloto }
$$

$A_{2}:$ Intervención de los investigadores en el aula

$A_{3}$ : Encuestas aplicadas a profesores de la asignatura y a estudiantes que ya la habían cursado

$A_{4}$ : Análisis del desempeño de los estudiantes en la solución de problemas de optimización en el examen final del curso.

\section{Actividades Relevantes}

Para el presente artículo se desarrollan y comentan a continuación aspectos destacados de las dos primeras actividades por cuanto su análisis aporta elementos importantes para le reflexión que aquí se propone.

\section{$\mathrm{A}_{1}$ : Prueba Piloto}

Con un curso formado por 23 estudiantes de Cálculo Diferencial se realizaron lecturas, ejercicios y talleres para incentivar el uso de estrategias en la solución de problemas de optimización. Al final se realizó una sesión de solución de problemas para la cual se recomendó el empleo de una rúbrica orientada a reforzar el empleo de estrategias heurísticas específicas: definir variables, distinguir entre elementos variables y constantes, escribir un plan de acción, ejecutarlo y revisar el proceso.

Las categorías analizadas en el estudio son: Estrategias Heurísticas Empleadas EE y Consistencia de la solución de los problemas CS. Los niveles de desempeño se establecieron con base en los criterios que se muestran en la tabla 1

\begin{tabular}{|c|c|c|c|}
\hline & A & B & C \\
\hline EE & Evidente & Parcial & Ausente \\
\hline CS & Total & Parcial & Ausente \\
\hline \multicolumn{4}{|c}{ Tabla 1. Categorías de Análisis }
\end{tabular}

Los resultados obtenidos se registran en la tabla 2

\begin{tabular}{|l|l|l|l|}
\hline & A & B & C \\
\hline
\end{tabular}

\begin{tabular}{|c|c|c|c|}
\hline EE & $36 \%$ & $28 \%$ & $36 \%$ \\
\hline CS & $18 \%$ & $59 \%$ & $23 \%$ \\
\hline \multicolumn{4}{|c|}{ Tabla 2. Resultados }
\end{tabular}

Análisis de los Resultados de $\mathrm{A}_{1}$

Sólo un $64 \%$ de los estudiantes dejó evidencias escritas de haber empleado alguna estrategia, lo cual no descarta que lo hayan hecho sin registrarlo en el papel. Sin embargo, se esperaba que, ante la insistencia en el tema, el porcentaje hubiese sido mayor. Por otra parte, hay un claro predominio de la presentación por parte de los estudiantes de soluciones incompletas o parciales a los problemas propuestos.

Es importante destacar que tres estudiantes presentaron Soluciones Consistentes de la mayoría de los problemas sin Utilizar explícitamente las Estrategias sugeridas en la rúbrica

$\mathrm{A}_{2}$ : Intervención de los investigadores en el aula

Los investigadores orientaron las clases durante el mes de octubre de 2013 con un grupo de 26 estudiantes de Cálculo Diferencial de segundo semestre del programa de Ingeniería Civil, que previamente habían aprobado la asignatura Matemáticas Básicas cuyo programa comprende elementos de Algebra, Geometría, Trigonometría, Geometría Analítica y teoría básica de funciones de una variable.

Antes de la llegada de los investigadores, bajo la orientación de la docente titular AA, se desarrollaron los conceptos de límites, continuidad, derivada, técnicas de derivación, funciones crecientes y decrecientes, criterios de la primera y la segunda derivada y análisis de gráficas.

La presencia de los investigadores estaba específicamente dirigida a orientar el trabajo con los problemas de optimización empleando solamente el tiempo asignado en el programa para hacerlo. Aunque todas las acciones emprendidas proporcionaron información relevante, para los propósitos del presente artículo se reseña brevemente una de las actividades que aportó más elementos para estudiar el comportamiento de los estudiantes.

Enunciado: Una recta pasa por el punto $(3,2)$ y forma con los ejes coordenados en el primer cuadrante, un triángulo rectángulo. Determinar la ecuación de la recta que en las condiciones dadas determina un triángulo de área mínima. 


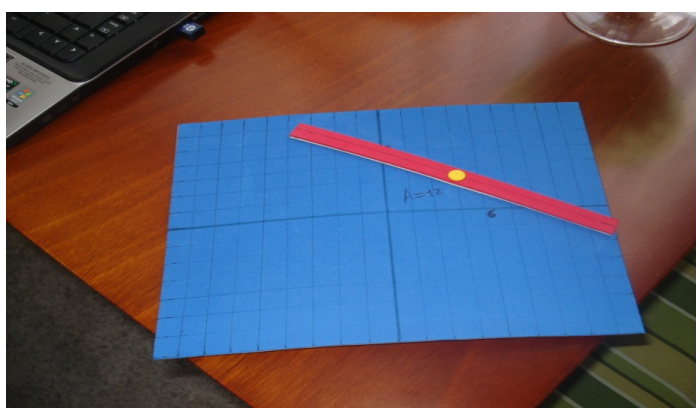

Fig 1. Material proporcionado a los estudiantes

1. Si la ecuación de la recta es $y=m x+b$, determinar las restricciones que deben cumplir los parámetros $\mathrm{m}$ y $\mathrm{b}$ para que el triángulo descrito por las condiciones se forme.

2. ¿Qué sucede si $b=2$ ?

3. ¿Es posible que $\mathrm{m}$ sea igual a 1 ?

4. Con el material de apoyo ubicar la recta en 5 posiciones diferentes, tomar las medidas y calcular el área de cada triángulo resultante registrando los resultados en una tabla.

Observar los registros de la tabla y conjeturar una solución.

5. Si la recta corta a los ejes en los puntos $(a, 0)$ y $(0, b)$ expresar el área del triángulo en función de a y b.

6. Encontrar una relación entre a y b de modo que el área del triángulo dependa solamente de $b$.

7. A partir de la función $A(b)$ encontrar los valores críticos y aplicar el criterio de la primera derivada para establecer los valores $(m$ y b ) de la recta $y=m x+b$.

8. Comparar los resultados obtenidos en el punto 7 con las estimaciones visuales y los datos de la tabla.

Desarrollo de la actividad: El profesor indicó a los estudiantes que el trabajo se debía realizar en forma colaborativa con la participación de todos los integrantes, sin repartir entre ellos los 8 puntos de la guía. Se organizaron libremente 9 grupos y en la primera parte los investigadores se limitaron a escuchar las discusiones de los estudiantes procurando no intervenir.

Se relacionan a continuación en forma cronológica algunas situaciones que se presentaron durante la clase por considerarlas significativas para el estudio. En su conjunto constituyen una reseña de la actividad realizada:

- Varios estudiantes intentan comenzar un proceso sin tener en cuenta instrucciones dadas por el profesor.

- Tardan más de 20 minutos para definir restricciones para la pendiente de la recta y el corte con el eje y (pregunta 1).
- En general, no asocian con claridad el signo de la pendiente de la recta con el hecho de ser creciente o decreciente.

- En dos de los grupos se interpreta erróneamente la pregunta 1 y se empeñan en buscar una relación entre $\mathrm{m}$ y $b$.

- Al tratar de ubicar la recta en posiciones distintas (pregunta 4), en 3 grupos se comete el error de sacar la aguja del punto $(3,2)$, ignorando una condición muy importante del problema.

- La tabla de datos que se pide en la pregunta 4, no se construye en forma adecuada en la mayoría de los grupos y las mediciones se hacen de forma demasiado imprecisa o se utilizan solamente números enteros.

- Los datos son registrados por varios grupos (4) en forma arbitraria sin una ordenación que permita detectar tendencias.

- Un estudiante sabe que en algún momento hay que derivar e insiste a sus compañeros en hacerlo, pero en ese momento todavía no tienen la función objetivo.

- El tiempo pasa y el progreso es lento.

- En un grupo trabaja una sola persona mientras los demás se distraen o aparentan observar.

- Un estudiante busca con insistencia la fórmula para el perímetro del triángulo y cuando se le pregunta como la aplicaría no responde y cambia el tema.

- Cuando van 70 minutos de trabajo ya hay varios estudiantes dispersos $(40 \%)$ que no aportan, los demás mantienen el interés.

- Gran dificultad para lograr que la función objetivo dependa solamente de b (pregunta 6). En un grupo proponen el uso de relaciones trigonométricas para expresar $\mathbf{a}$ en función de $\mathbf{b}$ pero no logran concretarlo.

- Al manipular el material real un estudiante afirma que la recta sólo puede ir hasta donde la base (azul) lo permite y esto limita su comprensión del problema.

- Luego de tener la función a optimizar, se observa en general, que los estudiantes conocen los criterios para optimizar una función, aunque operativamente tienen dificultades para aplicarlos.

- La mayor dificultad se registró para encontrar la función que relaciona el área del triángulo con la ordenada del punto $(0, b)$.

- El objetivo de encontrar la función, finalmente se logró concretar con la participación de los profesores enlazando aportes dispersos de los alumnos y a base de preguntas orientadoras. A continuación, se reconstruye parcialmente el mencionado trabajo colectivo en el que se destaca la participación activa de los estudiantes. 
Area $=\frac{\text { base } x \text { altura }}{2}=\frac{a . b}{2}$

La ecuación de la recta es $y=m x+b$

Si la recta pasa por los puntos $(a, 0) y(0, b)$ ¿que información puede obtenerse de esto?

Que la pendiente es $m=\frac{-b}{a}(*)$

El punto $(3,2)$ pertenece a la recta, entonces

$2=m \cdot 3+b \quad$ y sustituyendo $(*)$ en esta expresión,

se tiene $a=\frac{3 b}{b-2}$

Por lo tanto, la función para el Área es

$$
A(b)=\frac{3 b^{2}}{2(b-2)}
$$

- Luego de haber encontrado con cierta dificultad la función a optimizar, los estudiantes tenían la opción de utilizar una calculadora para hacer la gráfica, pero en general, no mostraron interés por obtener de ella información útil para la solución del problema.

En la pantalla se aprecia claramente la ubicación del mínimo y la restricción para la ordenada, $b>2$ se refleja también, pero ninguno de los estudiantes a quienes se les preguntó dio muestras de relacionar los procedimientos analíticos con la información que visualmente aparece. La presencia de la asíntota vertical tampoco fue vinculada con la imposibilidad de formar un triángulo cuando la recta que pasa por $(3,2)$ es paralela al eje $\mathrm{x}$.

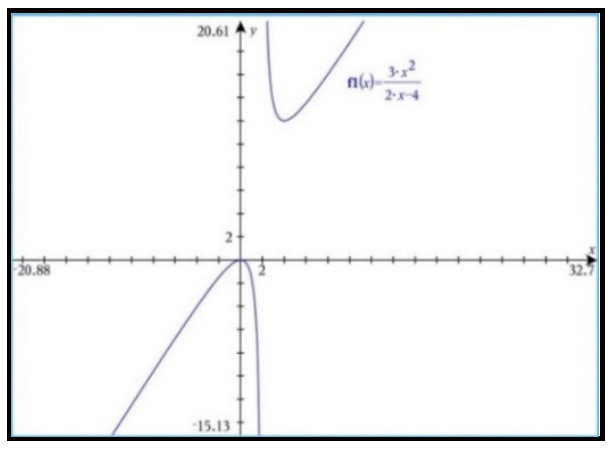

Fig 2. Gráfica de la función con calculadora TI N Spire CAS

\section{A. Análisis Cualitativo de los Resultados de $\boldsymbol{A}_{\mathbf{2}}$}

Las actividades realizadas durante el desarrollo del proyecto tanto con los estudiantes como con los profesores, así como la observación del desempeño de los alumnos en clase y el análisis de sus trabajos y evaluaciones permiten formular algunas conclusiones que pueden constituir un aporte para el mejoramiento de la Enseñanza de la Resolución de Problemas en la medida en que los investigadores pueden contrastar sus propias experiencias con las que se reportan en este estudio de caso.

Hay un predominio evidente de los procedimientos analíticos de carácter algebraico - algorítmico. En general, se observa que los estudiantes conocen los criterios de optimización y las técnicas de derivación, pero en este caso se trataba de encontrar primero la función sobre la cual se aplicarían.

Se registra un bajo nivel de aprovechamiento de la información proveniente de gráficas, tablas de datos u otras formas de representación, lo cual permite suponer que en la experiencia previa de los estudiantes no se hizo mucho énfasis en este importante aspecto. El tratamiento de datos numéricos es especialmente deficiente debido a que por no contar con una adecuada organización de la tabla en que los registraron, no pudieron realizar observaciones que aportaran a la solución.

En los grupos de estudiantes a los que se tuvo acceso se detecta un aprovechamiento muy limitado de la tecnología porque aun contando con calculadoras graficadoras, los estudiantes sólo las emplearon para hacer operaciones elementales que perfectamente se habrían podido efectuar con una calculadora corriente. Lo anterior puede reflejar que en los cursos previos no se han empleado adecuadamente estos recursos tecnológicos.

\section{NUEVAS PERSPECTIVAS PARA LA ENSEÑANZA DE LA RPM}

La Resolución de Problemas Matemáticos es un dominio de investigación y práctica en permanente evolución que fomenta el desarrollo de diferentes y a veces contradictorios enfoques sobre la adquisición del conocimiento matemático [17]. Incluye en su agenda análisis cognitivos, sociales y también los aspectos afectivos que influyen en el desarrollo de las competencias del estudiante para resolver problemas. Se considera relevante en este campo que ellos puedan utilizar eficientemente el conocimiento aprendido, que reflexionen sobre las estrategias de solución, formulen conjeturas y que cuenten con las herramientas comunicativas para expresar adecuadamente lo que piensan.

La característica más destacada de la agenda de investigación en RPM es que los temas tratados, las preguntas y los métodos han cambiado notablemente a través del tiempo. El desarrollo de métodos cuantitativos de análisis estadístico y el refinamiento de las metodologías cualitativas, así como el uso 
sistemático de tecnologías digitales no solo han mostrado nuevas rutas para representar y explorar las situaciones matemáticas a través del uso de modelos dinámicos, sino que la apropiación por parte de los estudiantes de estas herramientas proporciona nuevos rumbos a la investigación [17].

En [4] English y Sriraman presentan una revisión crítica de los principales enfoques sobre la RP con la intención de proyectar una mirada hacia el futuro. Reconocen inicialmente los autores el gran gran aporte de George Polya (1945) y consideran necesaria una actualización de este enfoque. En el mismo sentido, en 1992, Schoenfeld concluye que los intentos por enseñar a los estudiantes las estrategias de Polya, generalmente no han sido satisfactorios esencialmente porque éstas son más descriptivas que prescriptivas. Es decir, la mayoría son realmente sólo nombres para largas categorías de procesos en lugar de ser procesos bien definidos por sí mismos.

Se hace referencia en [4] a un reporte no muy alentador presentado por Silver (1985), en el cual muestra que incluso en casos donde fueron reportados aprendizajes exitosos empleando estrategias heurísticas, la transferencia no fue significativa y las mejoras se presentaron luego de largos y complejos cursos orientados por profesores expertos. Adicionalmente considera que los casos exitosos se deben más a los sólidos conocimientos de los alumnos que al empleo de las mencionadas estrategias.

Como confirmación de estas reflexiones, se presenta en [4] la conclusión de un trabajo realizado por Lester y Kehle en 2203, que da cuenta del siguiente hallazgo: " enseñar a los estudiantes estrategias heurísticas hace poco por mejorar su habilidad para resolver problemas matemáticos generales".

En efecto, las estrategias consideradas en forma aislada corren el riesgo de caer en lo instrumental y disminuir su impacto. Un error puede estar en la forma en que se trata la Resolución de Problemas en muchas aulas de clase en las que se enseñan primero los conceptos y luego se proponen problemas en que éstos se apliquen. Esta forma de enseñar deja la RP como un elemento aislado de las principales ideas $\mathrm{y}$ procesos matemáticos. Adicionalmente algunos profesores enseñan los conceptos y dejan a los estudiantes los problemas del libro texto como tarea. Es decir, en esta parte tan importante del quehacer matemático el profesor se hace a un lado y el alumno hace lo que puede.

Es necesario actualizar las preguntas con respecto a si las estrategias heurísticas son útiles o no; podemos preguntarnos por ejemplo ¿cómo puede integrarse la resolución de problemas con la comprensión de los conceptos esenciales del cálculo?, ¿cómo logar que una mejor comprensión de las estrategias heurísticas y su complemento con otras herramientas pueda incrementar su efectividad?

Un escenario bastante rico en posibilidades es el que se presenta con la implementación de la tecnología en el aula de clases pues convergen en ese entorno diferentes formas de representación y la implementación de heurísticas puede ser más ágil y estimulante para los estudiantes. Las posibilidades que ofrecen los computadores para crear realidades virtuales generan unas oportunidades educativas que no podían preverse hace una generación.

Particularmente para la comprensión de los conceptos esenciales del Cálculo Diferencial y para buscar nuevas formas de desarrollar la habilidad de los estudiantes en el planteamiento y RPM, los programas de Geometría Dinámica como Cabri o Geogebra, altamente interactivos y dotados de una sintaxis muy sencilla, muestran un panorama muy amplio de posibilidades para la enseñanza de las Matemáticas.

En efecto, para contratar el enfoque mostrado en la solución del problema de la recta que pasa por el punto $(3,2)$ que se analizó en la actividad $\mathrm{A}_{2}$ de la investigación reseñada, se presenta una solución aproximada obtenida con Geogebra.

Se representa en la pantalla la situación planteada en el problema, en ella se definió un deslizador para el punto $(0, \mathrm{~b})$ y se fijó el punto $(3,2)$.

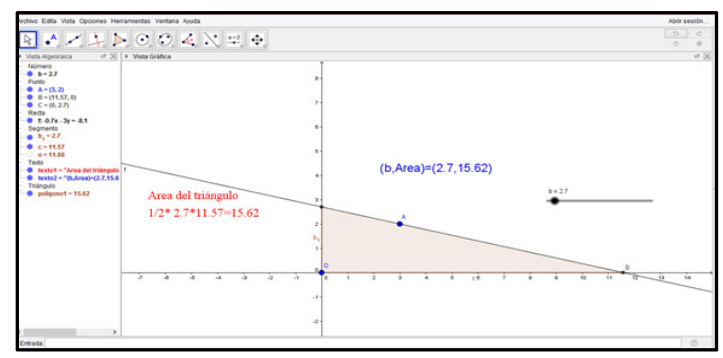

Fig. 3. Representación 1 con Geogebra

Igualmente se inserta un texto que va mostrando el área del triángulo en cada posición de la recta e incluso se forma la pareja ordenada $(b$, Area $)$

Al mover el deslizador b del punto (0,b), se observan diferentes momentos y se registran los valores del área en función de $\mathbf{b}$ aunque no se cuente con la expresión analítica de la función.

Específicamente puede registrarse el momento en que el Área es mínima observando como varía en función de la ordenada b. El Área mínima es de $12 u^{2}$ y se obtiene cuando $\mathrm{b}=4$, como se observa en la siguiente figura

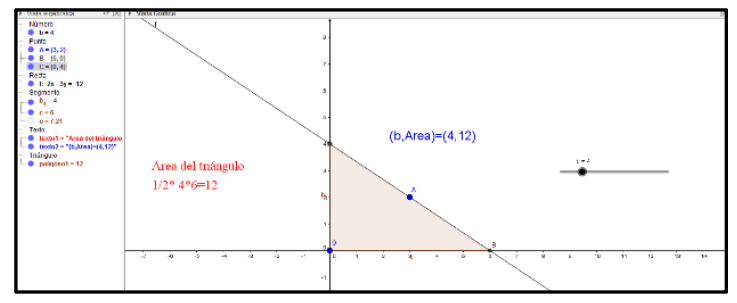

Fig. 4. Representación 2 con Geogebra

Observaciones importantes: 
Aunque en este caso no se llegó a la expresión analítica de la función, son los profesores quienes deben decidir sobre la importancia de obtenerla y orientar las tareas según su intención educativa. Es importante tener en cuenta que en muchos problemas sobre todo con datos reales, no es posible encontrar la función y una buena aproximación de la solución puede ser de utilidad práctica

Se requiere solamente una instrucción básica para utilizar este tipo de programas y particularmente para los jóvenes resulta más fácil familiarizarse con ellos. El profesor propone las actividades y problemas que deben resolverse y son los estudiantes quienes diseñan en el programa las representaciones necesarias para resolverlos. Para problemas más complejos la interacción del profesor con los estudiantes resulta muy productiva si se tiene en cuenta la multiplicidad de recursos disponibles. También se pueden graficar funciones, diseñar tablas de datos, representar en 3-D y, adicionalmente, participar en una comunidad de práctica a nivel internacional que comparte recursos y nuevas aplicaciones. Una ventaja adicional de Geogebra es que se trata de un software libre que funciona también en tabletas e incluso en teléfonos celulares.

Otra interesante ruta para el abordaje de la RPM se propone en [15] bajo el nombre de Perspectiva de Modelos y Modelación MMP desarrollada por Richard Lesh en 2003. Con el término Modelos hace referencia a las descripciones matemáticas de las situaciones en el entorno de los sistemas de práctica. La Modelación es un proceso de desarrollo de las representaciones para fines específicos. Por lo general implica una serie de pruebas, iteraciones y ciclos de revisión en los que las interpretaciones se ordenan y refinan teniendo en cuenta su carácter provisional. Pareciera ser ésta una tarea para profesionales, científicos y matemáticos, pero aquí el interés se centra en la necesidad de iniciar a los alumnos en estas formas de pensamiento mediante prácticas que les permiten comprender las relaciones entre el mundo real y los modelos matemáticos. Hay variedad de formas en que los estudiantes pueden participar en prácticas de modelado.

Se afirma en [15] que algunas teorías cognitivas parten de suponer que los seres humanos interpretan sus experiencias utilizando sistemas conceptuales internos que parecieran residir en la mente de los estudiantes. En MMP se considera que para seleccionar, filtrar, organizar y transformar la información en la solución de problemas complejos, se utiliza una gran variedad de medios que interactúan entre sí y que pueden ir desde la lengua hablada a los símbolos escritos, a los diagramas, a las metáforas o a las simulaciones por computador. El pensamiento es una forma de actividad mediada en la cual los significados se distribuyen a través de una gran variedad de medios que interactúan. En ese sentido "el modelado es una forma de alfabetización".

A medida que los estudiantes pasan por una serie de ciclos de modelado lo que se espera observar es la aparición de nuevas formas sistemáticas de pensar sobre la naturaleza de los objetos, las relaciones, las operaciones y los patrones o regularidades.
En [15] se reportan experiencias de modelado con estudiantes de diferentes niveles que pueden servir de referencia para el diseño de nuevas propuestas educativas. Como resultado de las investigaciones realizadas con este enfoque, ha surgido un nuevo libro titulado Más allá del Constructivismo: Perspectivas de Modelado y Modelación escrito por Lesh y Doerr en 2002. A partir del pensamiento de Vygotsky el libro hace hincapié en el papel de los instrumentos conceptuales que influyen en el pensamiento. Su importancia para estudiar el pensamiento matemático de los estudiantes es innegable.

En [6] se destaca la importancia del aprendizaje situado y la presencia de las matemáticas como parte de la cultura. Las relaciones entre el mundo real y las Matemáticas son particularmente relevantes, pero no es tarea sencilla acercar la complejidad de los fenómenos al aula, se requiere adecuar las prácticas al nivel de los estudiantes y no descuidar la comprensión de los conceptos esenciales de la disciplina.

La discusión sobre el rol de las aplicaciones y la Modelación en la enseñanza y aprendizaje de las Matemáticas es motivo de debate $\mathrm{y}$ se siguen formulando propuestas para su implementación pues existen claras evidencias que indican que hay una gran dificultad para transferir los conceptos matemáticos y aplicarlos directamente en situaciones que no hayan sido previamente matematizadas. La modelización puede ser vista desde el punto de vista educativo como un medio para aprender los conceptos o, como un fin.

Finalmente, en [14] en un artículo escrito por Martin A. Simon se describen las llamadas Trayectorias Hipotéticas de Aprendizaje THA como una estrategia para la planeación de las actividades a realizar en las clases de Matemáticas. Se trata de una construcción colectiva que establece unas metas específicas de aprendizaje de los estudiantes, diseña las actividades para promover dicho aprendizaje y elabora las hipótesis sobre este proceso. Muchos esfuerzos para mejorar la calidad de la educación Matemática en los Estados Unidos se han centrado en el diseño de las tareas porque se considera que una adecuada selección de las actividades y tareas que los estudiantes realizan juegan un papel clave en la eficacia de la enseñanza de las Matemáticas. Un aspecto importante es vincular las tareas con el concepto a aprender teniendo en cuenta que los ejercicios rutinarios poco contribuyen y que las preguntas retadoras y de contexto promueven la capacidad de pensar y de resolver problemas [14].

Una Trayectoria Hipotética de Aprendizaje comprende tres elementos esenciales: Unas metas u objetivos de aprendizaje, una hipótesis sobre la progresión del desarrollo del pensamiento de los alumnos y la secuencia de las tareas de instrucción. Dada la naturaleza hipotética e incierta de este proceso, el profesor está obligado a modificar sistemáticamente cada aspecto de la Trayectoria e irlo ajustando dependiendo de los resultados obtenidos y de las características de los estudiantes para los cuales se planifican las actividades. Esta metodología puede ser empleada para fines investigativos o de planificación. 


\section{CONCLUSIONES}

Muchas preguntas sobre la mejor forma de emplear la Resolución de Problemas en los cursos de Cálculo continúan abiertas, pero siempre habrá nuevas opciones para hacerlo. La presente reflexión muestra algunos caminos que pueden contribuir para el logro de una mejor comprensión de los conceptos matemáticos y como consecuencia de ello, un incremento notable de la habilidad de los estudiantes para resolver problemas.

Un uso moderado y sistemáticamente calculado de la tecnología puede ser una gran ayuda y producir un efecto estimulante para los estudiantes. Se recomienda enfatizar el trabajo con software especializado y el tratamiento de datos reales como los que brinda el entorno de Wolfram Alpha. La realización de talleres de manejo de recursos matemáticos digitales para profesores y estudiantes puede contribuir a la creación de una cultura matemática más dinámica y actual. Sin embargo, tampoco es bueno esperar que la tecnología resuelva todo porque si el estudiante se convierte solamente en espectador y usuario pasivo de lo que los dispositivos presentan, el efecto sería contrario porque podrían ver la ciencia como un video que pasa y se adquiere sin mediar esfuerzo, lo cual es opuesto a la naturaleza del conocimiento científico que es sistemático, organizado y muchas veces complejo [6].

Los acercamientos a la modelación que logren hacerse dependiendo del nivel académico de los estudiantes siempre brindarán experiencias motivantes y despertarán el interés de estudiantes y profesores. Como referencia para el lector se presentan en [6] ejemplos de temas que pueden ser abordados a través de procesos de Modelación empleando temas, fenómenos y objetos de la vida cotidiana en diferentes niveles de formación.

A la luz de estos nuevos desarrollos también deberían surgir herramientas para observar, analizar y evaluar los comportamientos de profesores y estudiantes en ambientes de aprendizaje con tecnología. En ese contexto surgirán nuevas heurísitcas como el "arrastre" o el uso de deslizadores en Geometría Dinámica [17] que muy probablemente no están caracterizadas.

Aunque algunos de los autores aquí referenciados exponen serias críticas a los enfoques basados en el uso de estrategias heurísticas, éstos siguen vigentes y muchos planes de estudio han sido diseñados con base en ellas. Valga decir, por ejemplo, que James Stewart autor de uno de los libros para la enseñanza del Cálculo de mayor impacto y difusión a nivel mundial, los utiliza y en la séptima edición del Cálculo de una Variable publicado en 2012, afirma: "creo que nadie ha mejorado mucho la estrategia de George Polya con sus cuatro etapas para resolver un problema". Es significativa esta afirmación si se tiene en cuenta que Polya las formuló por primera vez en 1945.
En la enseñanza de la Geometría tanto en la educación media como en la superior se perciben vacíos que se manifiestan especialmente cuando los estudiantes resuelven problemas. La Geometría Dinámica puede considerarse como un recurso valioso para tratar de enmendar tan notable deficiencia.

Desde el punto de vista motivacional es pertinente ir incrementando gradualmente la complejidad de los problemas que se proponen en clase. No es conveniente reforzar la concepción que muchos estudiantes tienen alrededor de ellos: según esta generalizada tendencia, los problemas son demasiado difíciles y es mejor dejar que otros los resuelvan. Es bueno que una persona experimente la agradable sensación de persistir hasta lograr comprender algo que en principio le resultaba difícil, pero que gracias a su esfuerzo logró resolver.

Las posibilidades de desarrollo de nuevas propuestas para desarrollar la habilidad de los estudiantes en la solución de problemas están abiertas y la problemática aquí planteada requiere acciones urgentes que logren aprovechar el gran potencial de los estudiantes que con grandes expectativas acuden a nuestras aulas. A ellos está dedicado este trabajo investigativo con un especial agradecimiento para quienes participaron y nos brindaron el privilegio de compartir con ellos y observar sus trabajos y acciones.

EL aprendizaje de los estudiantes tiene mucho que ver con su disposición para lograrlo y de su dedicación y esfuerzo. Depende también en gran medida de la calidad de la enseñanza y en ese sentido la interacción con el profesor durante las clases es fundamental y, por ahora, irreemplazable.

\section{REFERENCIAS}

[1] G. Polya, Cómo plantear y resolver problemas. México: Trillas, 2005.

[2] A. Schoenfeld, Mathematical Problem Solving. Orlando Florida: Academic Press, 1985.

[3] L.M. Santos Trigo, La Resolución de problemas matemáticos: fundamentos cognitivos, México: Trillas, 2007.

[4] 1. English, B. Sriraman. "Problem Solving for the $21^{\text {st }}$ Century". Theories in Mathematics Education:Seeking New Frontiers, Germany, Springer, 2010.

[5] R. Duval, Semiosis y pensamiento humano. Cali Universidad del Valle., 1999.

[6] W. Blum, P. Galbraith. "Modelling and Applications in Mathematics Education": The 14 th ICMI Study, Springer, New York, 2007.

[7] A. Schoenfeld. How We Think: A theory of Goal-Oriented Decision Making and its Educational Appplications. New York:Routledge, 2011 
[8] D. Tall, Advanced Mathematical Thinking, New York Kluwer Academic Publishers, , 2002.

[9] R. Duval . "Un tema crucial en la educación matemática: la habilidad para cambiar el registro de representación". La Gaceta de la RSME. Vol.91. pp. 143-168. 2006

[10] H. Gardner. La inteligencia reformulada: las inteligencias múltiples en el siglo XXI, Barcelona:Espasa Libros, , 2001.

[11] C. Azcárate, M. Camacho. "Sobre la investigación en Didáctica del Análisis Matemático", Boletín de la Asociación Matemática Venezolana, Vol X, No. 2, 2003.

[12] G. Polya, Matemáticas y Razonamiento Plausible, Madrid:Editorial Tecnos, , 1966

[13] L. Puig. "Presencia y ausencia de la resolución de problemas en la investigación y el currículo". Investigaciones en educación Matemáticas 12. SEIEM. 2008.

[14] D. Clements, J. Sarama. Mathematical Thinking and Learning. Special Issue: Hypothetical Learning Trajectories. Volumen 6, número 2,2004

[15] R. Lesh. Mathematical Teaching and Learning. Special Issue: Models and Modelling Perspective. Volumen 5, números 2-3, 2003

[16] L.M. Santos. “ La Resolución de problemas Matemáticos: Avances y perspectivas en la Construcción de una Agenda de Investigación y Práctica”. En Actas del Simposio de la Sociedad Española de Investigación en Educación Matemática. 2008

[17] L.M. Santos. "Problem Solving in Mathematics Education". Encyclopedia of Mathematics Education..Lerman (editor) 